\title{
VALIDITY STUDY OF ROUGH SURFACE SCATTERING MODELS
}

\author{
Floyd W. Millet and Karl F. Warnick \\ 1 Brigham Young University, Microwave Earth Remote Sensing Laboratory \\ $459 \mathrm{CB}$, Provo, UT 84602 \\ e-mail: milletf@et.byu.edu,warnick@ee.byu.edu
}

\begin{abstract}
We present a study of the regions of accuracy of various rough surface scattering models including the physical optics approximation and the small perturbation method. The accuracy of the models is compared for surfaces with power law and Gaussian power spectra. RMS error values between the exact method of moments solution and the models are shown over the entire parameter space studies. Results of the study also demonstrate a linear relationship between the accuracy of the methods and the moments of the surfaces.
\end{abstract}

\section{INTRODUCTION}

In the 1960's and 70's the small perturbation method (SPM) and physical optics (PO) approximations to electromagnetic scattering from a rough surface were developed. These approximations are simplified solutions to the complex interactions of electromagnetic waves with media in the low frequency and high frequency limits, respectively. Due to their longevity and simplicity of application, the PO and SPM approximations are well known and extensively used in the electromagnetic scattering communities. Since the models are derived for low frequency and high frequency approximations, there are limits to the range of surfaces for which they are valid.

In the last few decades other electromagnetic scattering models have been derived to improve the approximations and increase the range of surfaces over which the scattering models can be applied. The new generation of models generally reduce, at the lowest order to PO or SPM.

Limitations in the computational power available have caused most previous studies to a focus on the bistatic scattering from surfaces. A small number of surface types and incident angles have been used from which general patterns to determine the validity of the much larger parameter spaces have been inferred.

This study uses the increased calculation power now available for three important contributions. The first contribution is a more dense collection of points over the chosen parameter space. This provides a more complete picture of the accuracy of the models for the given parameters and allows for more accurate determinations of the regions of validity for the models. The second is to establish the relationship between the accuracy of the different models and the moments of the surfaces. The third contribution is a direct comparison of the difference in regions of accuracy between Gaussian and power law surfaces.

\section{ROUGH SURFACE SCATTERING}

We investigate the scattering of a transverse magnetic (TM) plane wave scattering from a perfectly conducting one-dimensional surface into two-dimensional space. The incident wave is time harmonic and traveling in the $\mathrm{x}-\mathrm{z}$ plane. We define the electromagnetic wavenumber as $k=2 \pi / \lambda$ and the electric field as

$$
\bar{E}=-\hat{y} E_{o} \exp \left(i k_{x} x-i k_{z} z\right) .
$$

where $k_{x}$ as the X-component of the incident electromagnetic wavenumber, $k_{x}=k \cos (\theta)$ and

$$
k_{z}=\sqrt{k^{2}-k_{x}^{2}} \operatorname{Im}\left(k_{z}\right)>0 .
$$

The convention used throughout this paper is that $k$ refers to the electromagnetic wavenumber and $K$ refers to surface wavenumbers. All surfaces in the study have a Gaussian height distribution.

\subsection{Gaussian Surfaces}

Surfaces defined by a Gaussian power spectrum are single-scale surfaces due to their band-limited spectrum. Because the attributes of such surfaces are well known, a number of studies have been conducted using such surfaces. Gaussian surfaces are completely defined statistically by the correlation length, $l$, and the surface height variance, $h^{2}$. The surface height power spectral density (PSD) is described by

$$
W(K)=\frac{l h^{2}}{(2 \sqrt{\pi})} \exp ^{\left(-K^{2} l^{2} / 4\right)}
$$

The parameter space for the Gaussian surfaces is described by the dimensionless parameters $k h$ and $k l$.

\subsection{Power Law Surfaces}

Surfaces described by a power law spectrum are representative of many surfaces found in nature. This type of surface is multi-scale due to the slow fall-off of the PSD 
for high wavenumbers. It is described by

$$
W(K)=h^{2}(p-1) K_{\min }^{(p-1)} K^{-p}, K>K_{\min }
$$

where the three parameters necessary to completely characterize the surface are $h^{2}$, the surface height variance, $K_{m i n}$, the minimum cutoff wave number, and $p$, the power of the spectrum.

Similar to the parameter space for the Gaussian surfaces, the parameter space is defined by two dimensionless variables, $k h$ and $k L$, where $L$ is analogous to the correlation length of the Gaussian, and defined by $L=\left(2 \pi / K_{\min }\right)$. For this study the power of the spectrum, $p=3$, which is an approximation of a spectrum of ocean surfaces.

\section{$3 \underline{\text { MODELS }}$}

The scattering models in this study are divided into two general types. Stochastic models use formulae derived from the statistical properties of the surface to calculate average scattering from the surface. Deterministic models compute the backscatter for a single surface and are averaged using Monte Carlo over many realizations to obtain stochasitc results.

The stochastic models in this study are geometrical optics (GO), physical optics (PO), the first two expansion terms of the small perturbation method (SPM1, SPM2), and the first three orders of the small slope approximation (SSA1, SSA2, SSA3). The Monte Carlo methods investigated are iterated physical optics (ITPO) and Monte Carlo physical optics (MCPO).

A brief review of the derivation of the various models is presented here. A more detailed derivation can be found in the references.

\subsection{Physical Optics}

Physical optics (PO) is the oldest and most well-known rough surface scattering model. It is based on the premise that the surface can be modeled as a series of tangent planes which approximate the actual surface. Using the tangent plane approximation, the scattered electric field can be written as

$$
\vec{E}^{s}=\frac{k \eta_{0}}{4} \int_{S} \vec{J}_{s}\left(\rho^{\prime}\right) g\left(\rho, \rho^{\prime}\right) \mathrm{d} S^{\prime}
$$

where

$$
\begin{aligned}
J_{s} & =\hat{n} \times\left(H^{i}+H^{s}\right) \\
& =2 \hat{n} \times H^{i}
\end{aligned}
$$

and $g\left(\rho, \rho^{\prime}\right)$ is the Green's function for the appropriate geometry and $J_{s}\left(\rho^{\prime}\right)$ is the surface current. The normalized backscatter coefficient can then be calculated by

$\sigma^{0}=\frac{1}{L^{2}}\left\langle\frac{4 \pi r^{2} \vec{E} \vec{E}^{*}}{E_{0}^{2}}\right\rangle$

$$
=\frac{k \cos ^{2}(\theta)}{\pi} \iint \mathrm{d} x \mathrm{~d} x^{\prime} \mathrm{e}^{i k_{b}}\left\langle\mathrm{e}^{i 2 k_{x}\left[\eta(x)-\eta\left(x^{\prime}\right)\right.}(\oint)\right.
$$

where $k_{b}=2 k \sin (\theta)$ is the Bragg wave number. Further simplifications and algebra lead to a backscatter coefficient of ${ }^{1}$

$\sigma^{0}=\frac{k \cos ^{2}(\theta)}{\pi} \int_{-L}^{L} \mathrm{~d} x\left(1-\frac{|x|}{L}\right) \mathrm{e}^{i k_{b} x} \mathrm{e}^{-\chi^{2}[1-C(x)]}$

where $C(x)$ is the correlation coefficient, $h^{2}$ is the surface height variance, and $\chi=2 h k_{z}$. From this equation it can be seen that the backscatter coefficient can be expressed in terms of the known stochastic properties of the surface, $C(x)$ and $h$.

\subsection{Geometrical Optics}

Geometrical optics (GO) is the infinite frequency limit of the PO approximation. If the slope variance of the surface is finite we can approximate the correlation function $C(x)$ as $1-C^{\prime \prime}(0) x^{2} / 2$, and $\mathrm{Eq}$. (10) reduces to

$$
\sigma^{0}=\frac{k \cos ^{2}(\theta)}{\pi} \exp \left[\frac{-k_{b}^{2}}{2 \pi \chi\left|C^{\prime \prime}(0)\right|}\right]
$$

where $\left|C^{\prime \prime}(0)\right|$ is the normalized slope variance.

\subsection{Iterated Physical Optics}

The normalized backscatter is calculated from iterated physical optics model (ITPO) using the magnetic field integral equation,

$$
\begin{aligned}
\hat{n} \times \vec{H}^{i}(\rho) & =\left(/ 2 I-\nabla \times \int g\left(\rho, \rho^{\prime}\right)\right) \vec{J}_{s}\left(\rho^{\prime}\right) \\
& =(I-M) J_{s}
\end{aligned}
$$

where $M$ denotes the integral operator in Eq. (13). An exact solution could by calculated by multiplying both sides of the previous equation by $(I-M)^{-1}$. For the ITPO model, we expand $(I-M)^{-1}$ in a series as

$$
(I-M)^{-1}=I+M+M^{2}+M^{3}+\ldots
$$

If we assume that $M$ is small, then the first two terms are sufficient to approximate the series and the surface current becomes

$$
J_{s} \approx(I+M)\left(2 \hat{n} \times H^{i}\right) .
$$

From the surface current the scattered fields can be easily calculated.

\subsection{Monte Carlo Physical Optics}

The Monte Carlo Physical Optics (MCPO) model is derived directly from Eq. (5). From the tangent plane approximation, the surface current can be approximated as

$$
\vec{J}_{s}=2 \hat{n} \times \vec{H}_{i} .
$$


Given the surface profile, the normal vectors are easily calculated, and $J_{s}$ is substituted back into Eq. (5).

\subsection{Small Perturbation Method}

While the PO approximation is used primarily with high frequency incident wave, the small perturbation method (SPM) is the classical method that is typically used for low frequency modeling. The small perturbation method can be derived from extinction theorem,

$$
E^{s}=\int J_{s}\left(\rho^{\prime}\right) g\left(\rho, \rho^{\prime}\right) \mathrm{d} S,
$$

where $g\left(\rho, \rho^{\prime}\right)$ is expressed as the Weyl plane-wave expansion of the Green's function and the normal derivative of the electric field on the surface is described by

$$
J_{s}=C \mathrm{e}^{i k_{x} x} f(x) .
$$

The scattered electric field is calculated by expanding $f(x)$ and $\mathrm{e}^{i k_{x} x}$ in a power series, and collecting like terms. The classic form equation for the SPM is

$$
\sigma^{0}=\frac{4 k_{z}^{4}}{k} W\left(2 k_{x}\right)
$$

Due to the complexity of the second term (SSA2), it is not shown. ${ }^{2}$

\subsection{Small Slope Approximation}

The small slope approximation is another perturbation method where the scattering amplitude, $T$, is expressed as

$$
T=-\left.\frac{1}{2 \pi} \int \mathrm{d} x \Phi \mathrm{e}^{i 2\left(k_{x} x+k_{z} z\right)}\right|_{z=\eta(x)}
$$

where $\Phi$ is expanded in a power series. As with SPM, like terms are grouped together with each successive order of the model giving a more accurate result. The first order SSA (SSA1) model gives the same result as the PO model in Eq.(10). Each successive addition to the model includes another term. The second order approximation of the SSA model (SSA2) is

$$
\begin{array}{r}
\sigma_{S S A 2}^{0}=\sigma_{S S A 1}^{0}+\frac{k_{z}^{2}}{\pi} \exp \left(-\chi^{2}\right) \\
\times \operatorname{Re}\left\{-J^{*} \int \mathrm{d} x e^{i k_{b}} B(x)\right. \\
\left.+\int \mathrm{d} x I(x) \int \mathrm{d} K \exp ^{i K x} W(K) g^{*}(K)\right\} .
\end{array}
$$

where

$$
\begin{aligned}
I & =\exp ^{i k_{b} x} e^{\chi^{2} C(x)} \\
J & =\int \mathrm{d} K W(K) g(K) \\
g(K) & =k\left(\sqrt{k^{2}-\left(K-k_{x}\right)^{2}}\right. \\
& \left.+\sqrt{k^{2}-\left(k_{x}-K\right)^{2}}-2 k_{z}\right)
\end{aligned}
$$

Similarly, the third order SSA model (SSA3) is an addition to SSA2. Due to the complexity of the equation it is not presented here ${ }^{3} .4$

\section{NUMERICAL IMPLEMENTATION}

The models studied in this paper are compared against an exact method of moments (MOM) solution of the scattering problem. ${ }^{5}$

\subsection{Method of Moments}

The method of moments solution to scattering from a rough surface for a TM wave begins with the integral solution to the wave equation

$$
E^{i}=\frac{k \eta_{0}}{4} \int_{S} J_{s}\left(\rho^{\prime}\right) H_{0}^{(2)}\left(k\left|\rho-\rho^{\prime}\right|\right) \mathrm{d} S^{\prime}
$$

In matrix form this can be rewritten as

$$
\begin{gathered}
\left(\begin{array}{c}
E^{i}\left(\rho_{1}\right) \\
E^{i}\left(\rho_{2}\right) \\
\vdots \\
E^{i}\left(\rho_{N}\right)
\end{array}\right)= \\
\left(\begin{array}{cccc}
A_{11} & A_{12} & \cdots & A_{1 N} \\
A_{21} & A_{22} & \cdots & A_{2 N} \\
\vdots & \vdots & \ddots & \vdots \\
A_{N 1} & A_{N 2} & \cdots & A_{N N}
\end{array}\right) \quad\left(\begin{array}{c}
J_{s}\left(\rho_{1}\right) \\
J_{s}\left(\rho_{2}\right) \\
\vdots \\
J_{s}\left(\rho_{N}\right)
\end{array}\right)
\end{gathered}
$$

where

$A_{m n} \approx\left\{\begin{array}{cc}\frac{k \eta_{0}}{4} \Delta S H_{0}^{(2)}\left(k\left|p_{n}-p_{m}\right|\right) & m \neq n \\ \frac{k \eta_{0}}{4} \Delta S\left[1-j \frac{2}{\pi} \ln \left(\frac{\gamma k \Delta S}{4 \mathrm{e}}\right)\right] & m=n\end{array}\right.$

and $\mathrm{e}=2.718 \ldots$ and $\gamma=1.781 \ldots$ By inverting $A$, the source current, $J_{s}$ can be solved for, from which the scattered electric fields can be calculated.

\subsection{Electric Field Tapering}

To eliminate the edge effects that accompany finite length surfaces, a Gaussian windowing function, $G(x)$ has been applied to the incident electric field. Acceptable angular resolution and stability can be obtained with the taper function ${ }^{6}$

$$
G(x)=\frac{\mathrm{e}^{-\left(x-x_{m}\right)^{2}}}{g^{2}}
$$

where $g=L / 12, L$ is the length of the surface, and $x_{m}$ is the midpoint of the surface.

\subsection{Monte Carlo Convergence Study}

Monte Carlo models require a large number of instantiations of surfaces to calculate and accurate average 
backscatter value. In addition to the monte carlo calculations required for the exact MOM solution, the Monte Carlo models included in this study are Monte Carlo Physical Optics (MCPO) and the Iterated Physical Optics (ITPO). The instantiations used to compute the backscatter for the exact solution were also used for these two models. The Monte Carlo method lead to a certain amount of variability from the true average that decreases with an increase in instantiations. To determine the convergence, 10000 instances of the surface were created and used to calculate the backscatter. The results, seen in Fig. 1, show that the errors decrease as the square-root of the number of instantiations. We have chosen to use 50 surfaces for our Monte Carlo simulations, corresponding to an error of $-4.5 \mathrm{~dB}$.

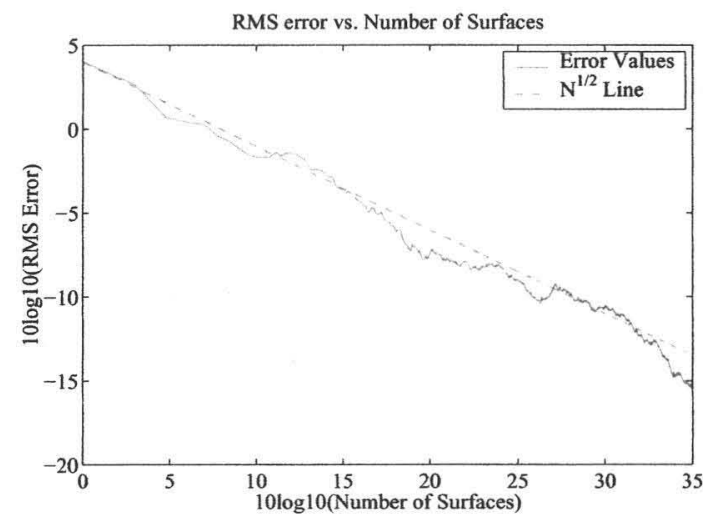

Figure 1: The RMS error values for successive numbers of surface instantiations for Monte Carlo averaging of the scattering coefficient.

\section{RESULTS}

This paper investigates the backscattered returns for surfaces derived from Gaussian and power law power spectra. The results have been divided into three sections based on incidence angle. The near-nadir range is defined as the region between $0^{\circ}$ and $5^{\circ}$. The mediumangle and large-angle ranges are defined as $5-20^{\circ}$ and $20-60^{\circ}$, respectively.

To compute the error metric used in this study, the difference between the exact MOM value and the model value was calculated at $0.3^{\circ}$ increments. The RMS error values, in decibels, for each angular region were computed and are shown in the error plots. The continuum of error values are in shown in grey-scale.

Models based on PO approximations have been shown to be more accurate at near-nadir incidence an- gles, while the SPM model is know to have a region of validity at larger incidence angles.

A side-by-side comparison of the regions of validity for Gaussian and power law surfaces is also available. Further, we demonstrate that the accuracy of the models can be described by a linear correlation with the wave slope variance of the surface.

\subsection{Near-Nadir Angles}

The physical optics approximation for rough surface scattering yields itself well to analysis for surfaces that are "smooth" with respect to the electromagnetic wavelength. For Gaussian surfaces, this can be described by a relationship with the curvature of the surface. However, application of the physical optics model to more complex surfaces, such as power law surfaces, is not well documented due to the presence of waves ranging from many electromagnetic wavelengths to waves with wavenumbers much larger than the EM wavenumber, which cause roughness at all spatial frequencies.

\subsubsection{Gaussian Surfaces}

The smoothness criteria for the validity of the PO approximation for Gaussian surfaces is widely accepted as the RMS wave slope of the surface. The slope of the surface can be calculated as the second moment of the power spectrum

$$
\begin{aligned}
s & =\sqrt{\int \mathrm{d} K K^{2} W(K)} \\
& =\frac{\sqrt{2} h}{l} .
\end{aligned}
$$

When the slope of the surface is expressed in the parameter space described previously, it can be see the that the isoslope lines will be linear and pass through the origin. Figure 2, shows that the regions of validity show a distinct linear correlation with the slope values for Gaussian surfaces.

\subsubsection{Power Law Surfaces}

Similar to Gaussian surfaces, the accuracy of power law surfaces can be accurately described by a smoothness criteria related to the curvature statistics of the surface. The variance of curvature, $c^{2}$, of a surface can be calculated as the fourth moment of the power spectrum, $W(K)$,

$$
c^{2}=\int \mathrm{d} K K^{4} W(K)
$$

Surfaces with a power law spectra are described by $W(K) \propto K^{-p}$, as seen in Eq. (4). For cases where $p<3$, the result of the curvature integral will be infinite. Warnick and Arnold ${ }^{7}$ show that the PO model 
has a built-in smoothing effect that ignores the waves whose wavelength and amplitude are of an order of magnitude smaller than the electromagnetic wavelength, effectively truncating the power spectrum and creating a surface with a finite curvature. The truncated surface can be expressed as

$$
c^{2}=\int_{(p i / \sqrt{2}) \sigma}^{(p i / \sqrt{2}) \sigma} \mathrm{d} K K^{4} W(K) .
$$

The radius of curvature corresponding to two standard deviations of the curvature is described by $r_{\sigma}=1 / 2 c$. Theory provided by Warnick and Arnold ${ }^{7}$ indicates that $\lambda_{e m} \ll r_{\sigma}$ for PO to be valid. Due to the relationship of the PO model with the remaining models in this study, the correlation between the curvature of the truncated surface and model accuracy extends to all the models in the study, with the exception of SPM.

\subsubsection{Coherent Scattering}
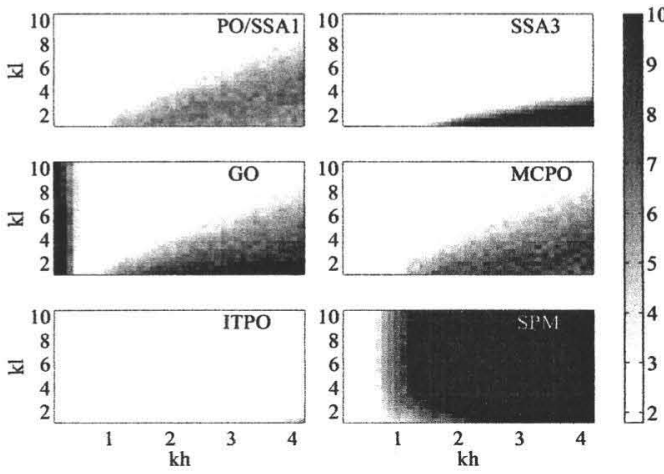

Figure 2: RMS error values for near-nadir incidence angles of a Gaussian surface.

Unexpected error values can be seen for small $k h$ values in many of the models. At the near-nadir incidence angles the backscatter for smooth surfaces, where $k h$ is small, is dominated by the coherent component. For all but the GO model, the coherent return has been subtracted from the total backscatter return. Though the incoherent backscatter values were very small for the models and the exact solution, due to computer precision, the order of magnitude of the values was large enough to show significant relative errors on a decibel scale. For the GO model, the coherent and incoherent returns are not readily separable, thus both are included in the calculations leading to large error values where there is a significant coherent component.

The SPM model is unique among the models studied in the expected regions of validity. It is well known that

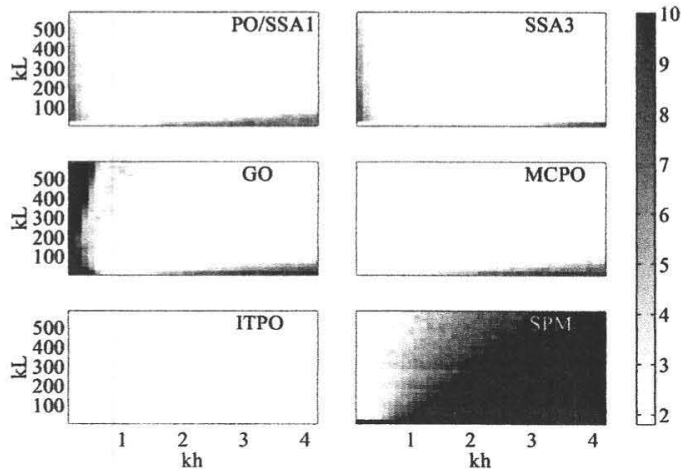

Figure 3: RMS error values for near-nadir incidence angles of a power law surface.

the SPM model is more accurate for larger incidence angles. Thus for the near-nadir region it can be seen that the SPM model demonstrates large errors across most of the parameter space, as seen in fig. 3 , with a small region of validity for small $k h$ values.

\subsection{Medium Incidence Angles}

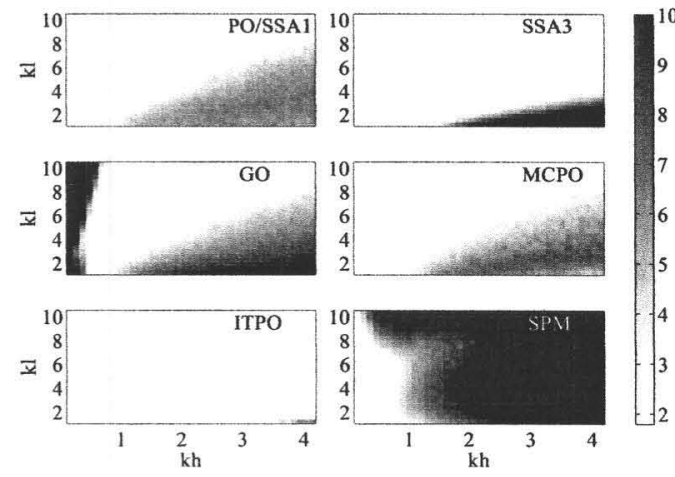

Figure 4: RMS error values for medium-range incidence angles of a Gaussian surface.

The error values for the medium-range incidence angles are very similar to those seen at the near-nadir angles. One noticeable improvement that can be seen for power law surfaces is the lack of error values at small $k h$ values for the PO and SSA3 models, due to an absence of coherent backscatter in the medium-range angles. The move away from nadir has reduced the errors associated with the coherent scattering seen for small $k \mathrm{~h}$ values. The medium range angles also show the transi- 


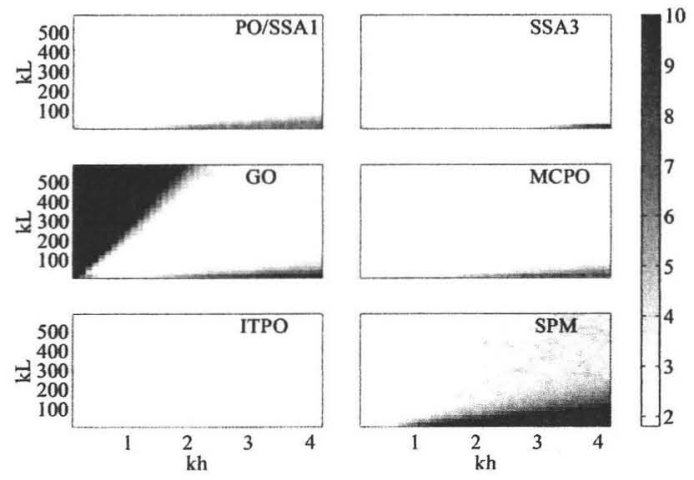

Figure 5: RMS error values for medium-range incidence angles of a power law surface.

tion between the accuracy of the GO model at near-nadir incidence angles, to the increasing accuracy of the SPM at large incidence angles. As with the near-nadir region, the accuracy of the Gaussian and power law surfaces are determined by a linear relationship with the moments of the surface, as seen in Fig 4 and Fig 5.

\subsection{Large Incidence Angles}

The models in this study show a strong angular dependence when calculating the backscatter for the Gaussian surfaces, as seen in Fig 6 and Fig 7. The RMS error for all models, with the exception of MCPO, is significantly greater for the large incidence angles. Of interest, are the large error values shown by the SPM model for the Gaussian surfaces. It can also be seen for large $k l$ and small $k h$, that all models show significant error.

For the large incidence angles, the power law surfaces show a general degradation for the models based on the tangent plane approximation, though SSA3 and ITPO demonstrate a robustness that extends beyond the angular range of the other PO base models. The GO model degrades over the almost the entire parameter space for both surface types.

\section{SUMMARY}

This paper presents a study of the regions of validity of various rough surface scattering models for surfaces with a Gaussian and power law power spectra. One of the contributions included in this papers is a presentation of the continuum of error values for each of the models over a complete parameter space. This presentation allows for individual determination of required accuracy for a broad range of applications. We have also shown a linear correlation between the moments of both surface types and the
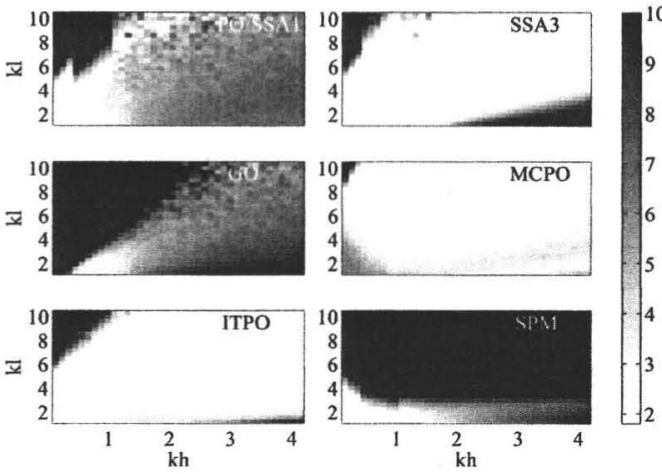

Figure 6: RMS error values for large incidence angles of a Gaussian surface.
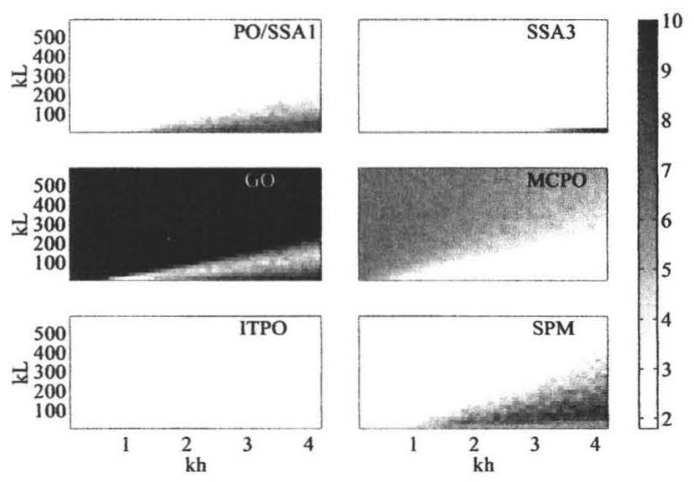

Figure 7: RMS error values for large incidence angles of a power law surface.

accuracy of the models. For Gaussian surfaces, isolines for accuracy have a one-to-one correspondence with the surface slope. For power law surfaces this relationship is shown to exist with the radius of curvature of the surface.

The accuracy of the models was also shown to have an angular dependence. As has been found in previous studies, the models derived from a tangent plane approximation were generally more accurate for small incidence angles. The angular dependence of error was especially pronounced for the Gaussian surfaces. The models the retain the most accuracy at large incidence angles were the iterated physical optics model and the third order small slope approximation.

Of interest was the relationship between the classic scattering theories, PO and SPM. It is commonly held that the regions of validity of SPM and PO are disjoint over a given parameter space, with $\mathrm{PO}$ accu- 
rate at smaller incidence angles and with smoother surfaces, and SPM more accurate at larger angles and with smaller surfaces. Though there are indications that for power law surfaces at incidence angles larger than $60^{\circ}$, the SPM model could be more accurate than PO, this study showed that for the surface types, parameter space and angular regions considered there was no case where the SPM method performed better than the PO method.

\section{References}

[1] A. D. Greenwood, "Azimuth modulation of the radar backscatter at near-normal incidence," Ph.D. dissertation, Brigham Young University, 1995.

[2] E. I. Thorsos and D. R. Jackson, "The validity of the perturbation approximation for rough surface scattering using a gaussign roughness spectrum," Journal of the Acoustical Society of America, vol. 86, no. 1, pp. 261-277, July 1989.

[3] S. L. Broschat and E. I. Thorsos, "An investigation of the small slope approximation for scattering from rough surfaces. part II. numerical studies," Journal of the Acoustical Society of America, vol. 101, no. 5, pp. 2615-2625, May 1997.

[4] E. I. Thorsos and S. L. Broschat, "An investigation of the small slope approximation for scattering from rough surfaces. part I. theory," Journal of the Acoustical Society of America, vol. 97, no. 4, pp. 2082-2093, April 1995.

[5] M. N. O. Sadiku, Numerical Techniques in Electromagnetics, second edition ed. CRC Press, Boca Raton, 201.

[6] R. Axline and A. K. Fung, "Numerical computation of scattering from a perfectly conducting random surface," IEEE Transactions on Antennas and Propagation, vol. AP26, no. 3, pp. $48-488$, May 1978.

[7] K. F. Warnick and D. V. Arnold, "Generalization of the goemetrical-optics scattering limit for a rough conducting surface," Journal of the Optical Society of America, vol. 15 , no. 9, pp. 2355-2361, September 1998. 